\title{
New Physics at 1TeV?
}

\author{
S.I. Godunov ${ }^{1,2, \star}$ \\ ${ }^{1}$ Institute for Theoretical and Experimental Physics, 117218, Moscow, Russia \\ ${ }^{2}$ Novosibirsk State University, 630090, Novosibirsk, Russia
}

\begin{abstract}
We consider the extension of the Standard Model with an extra scalar $S$ which decays can be responsible for the diphoton excess with invariant mass $\sim 750 \mathrm{GeV}$ observed at the $13 \mathrm{TeV}$ LHC run. Two scenarios of $S$ production are considered: gluon fusion through a loop of heavy isosinglet quark(s) and photon fusion through a loop of heavy isosinglet leptons. In the second case many heavy leptons are needed or/ and they should have large electric charges in order to reproduce experimental data on $\sigma_{p p \rightarrow S X} \cdot \operatorname{Br}(S \rightarrow \gamma \gamma)$.
\end{abstract}

\section{Introduction}

ATLAS and CMS collaborations recently announced a small enhancement over smooth background of two photon events with invariant mass $750 \mathrm{GeV}[1,2]$. Though statistical significance of this enhancement is not large (within 3 standard deviations), it induced a whole bunch of theoretical papers devoted to its interpretation. The reason for this explosive activity is clear: maybe the Standard Model is changed at one $\mathrm{TeV}$ scale, and we are witnessing the first sign of New Physics.

Let us suppose that the observed enhancement is due to the $\gamma \gamma$ decay of a new particle. Then it should be a boson with spin different from one: the simplest possibility is a scalar particle $S$ with $m_{S}=750 \mathrm{GeV}$. Since it decays to photons, it should be neutral and colorless, therefore in $p p$-collisions it can be produced in gluon-gluon fusion through the loop of colored particles and in photon-photon fusion through the loop of charged particles. Let us suppose that $S$ decays to these particles are kinematically forbidden, otherwise $\operatorname{Br}(S \rightarrow \gamma \gamma)$ reduces significantly which makes $S \rightarrow \gamma \gamma$ decays unobservable at the LHC.

We suppose that the particles propagating in the loop are Dirac fermions, so they have tree level masses, and that they are $S U(2)_{\mathrm{L}}$ singlets. Nonzero hypercharges provide couplings of these particles with photon and $Z$-boson. These particles can be quark(s) (color triplets) $T_{i}$ or lepton(s) (color singlets) $L_{i}$. They couple with $S$ by Yukawa interactions with coupling constants $\lambda_{T}^{i}$ and $\lambda_{L}^{i}$ correspondingly.

This talk is based on the paper [3] written in collaboration with A.N. Rozanov, M.I. Vysotsky, and E.V. Zhemchugov.

^e-mail: sgodunov@itep.ru 


\section{Quarkophilic $S$}

In the case of one heavy quark $T$ the following terms should be added to the Standard Model lagrangian:

$$
\Delta \mathcal{L}=\frac{1}{2}\left(\partial_{\mu} S\right)^{2}-\frac{1}{2} m_{S}^{2} S^{2}+\bar{T} \gamma_{\mu}\left(\partial_{\mu}-\frac{i}{2} g_{S} A_{\mu}^{i} \lambda_{i}-i g^{\prime} \frac{Y_{T}}{2} B_{\mu}\right) T+m_{T} \bar{T} T+\lambda_{T} \bar{T} T S,
$$

where $A_{\mu}^{i}$ and $B_{\mu}$ are gluon and $U(1)$ gauge fields respectively, and $\lambda_{i}$ are Gell-Mann matrices. $S$ coupling with gluons is generated by the $T$-quark loop:

$$
\begin{gathered}
M_{g g}=\frac{\alpha_{s}}{6 \pi} \frac{\lambda_{T}}{m_{T}} F(\beta) G_{\mu \nu}^{(1)} G_{\mu \nu}^{(2)} S, \\
\beta=\left(\frac{2 m_{T}}{m_{S}}\right)^{2}, F(\beta)=\frac{3}{2} \beta\left[1-(\beta-1) \arctan ^{2} \frac{1}{\sqrt{\beta-1}}\right] .
\end{gathered}
$$

Inclusive cross section of $S$ production in $p p$ collision at the LHC through gluon fusion is given by:

$$
\sigma_{p p \rightarrow S X}=\left.\frac{\alpha_{s}^{2}}{576 \pi}\left(\frac{\lambda_{T}}{m_{T}}\right)^{2}|F(\beta)|^{2} m_{S}^{2} \frac{d L_{g g}}{d \hat{s}}\right|_{\hat{s}=m_{S}^{2}},
$$

where the so-called gluon-gluon luminosity is given by the integral over gluon distributions:

$$
\frac{d L_{g g}}{d \hat{s}}=\frac{1}{s} \int_{\ln \sqrt{\tau_{0}}}^{-\ln \sqrt{\tau_{0}}} g\left(\sqrt{\tau_{0}} \mathrm{e}^{y}, Q^{2}\right) g\left(\sqrt{\tau_{0}} \mathrm{e}^{-y}, Q^{2}\right) d y,
$$

$\tau_{0}=\hat{s} / s, s=(13 \mathrm{TeV})^{2}$, and we use $Q^{2}=m_{S}^{2}$. Integrating gluon distributions from [4] for $\sqrt{\hat{s}}=$ $750 \mathrm{GeV}, \sqrt{s}=13 \mathrm{TeV}$, we get $d L_{g g} / d \hat{s} \approx 4.0 \mathrm{nb}, m_{S}^{2} d L_{g g} / d \hat{s} \approx(1 / 0.69 \mathrm{nb}) \cdot 4.0 \mathrm{nb} \approx 5.8$. In order to take into account gluon loop corrections, (4) should be multiplied by the so-called $K$-factor which is close to 2 for $\sqrt{s}=13 \mathrm{TeV}$, according to [5] (see also Fig. 2 in [6]).

In this way for $m_{T}=m_{S}$ and $\lambda_{T}=1$, substituting $\alpha_{S}\left(m_{S}\right)=0.090$, we obtain $\sigma_{p p \rightarrow S X} \approx 41 \mathrm{fb}$, which should be multiplied by $\operatorname{Br}(S \rightarrow \gamma \gamma)$ in order to be compared with experimental observations [1, 2]. Total width of $S$ is dominated by the $S \rightarrow g g$ decay, and from (2) we get:

$$
\Gamma_{S \rightarrow g g}=\left(\frac{\alpha_{s}}{6 \pi}\right)^{2} \cdot 8 \frac{m_{S}^{3} \lambda_{T}^{2}}{16 \pi m_{T}^{2}}|F(\beta)|^{2} \approx 3.1 \mathrm{MeV} .
$$

Thus for the models we consider, the width of $S$ should be much smaller than $45 \mathrm{GeV}$ which is preferred by the ATLAS data. Let us note that CMS data prefer narrow $S$; see also [7].

$T$-quark loop contributes to $S \rightarrow \gamma \gamma$ decay as well. The corresponding matrix element equals

$$
M_{\gamma \gamma}=\frac{\alpha}{3 \pi} \frac{\lambda_{T}}{m_{T}} F(\beta) F_{\mu \nu}^{(1)} F_{\mu \nu}^{(2)} \cdot 3_{\mathrm{c}} Q_{T}^{2},
$$

where the factor $3_{c}$ corresponds to the three colors, and $Q_{T}$ is the $T$-quark electric charge. For $\gamma \gamma$ width we get:

$$
\Gamma_{S \rightarrow \gamma \gamma}=\left(\frac{\alpha}{3 \pi}\right)^{2}\left(3_{\mathrm{c}} Q_{T}^{2}\right)^{2} \frac{m_{S}^{3} \lambda_{T}^{2}}{16 \pi m_{T}^{2}}|F(\beta)|^{2} \approx 22 \mathrm{keV},
$$




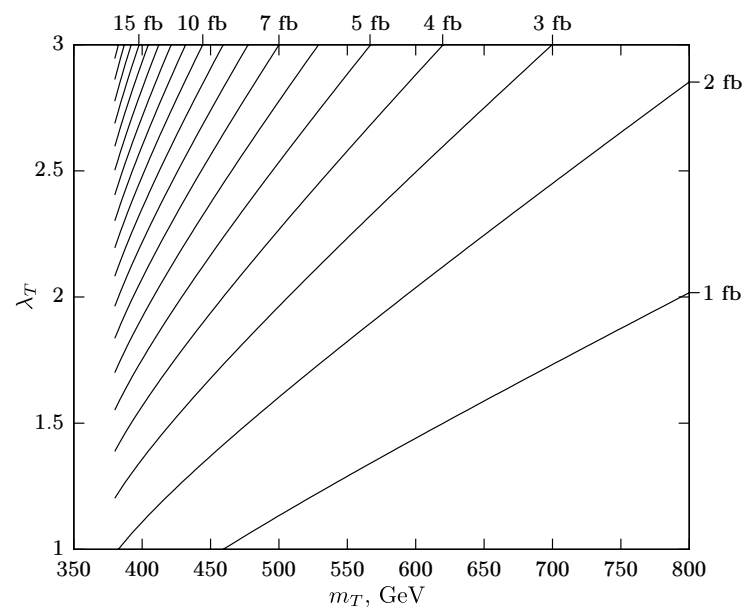

Figure 1. Contour plot of $\sigma_{p p \rightarrow S X} \cdot \operatorname{Br}(S \rightarrow \gamma \gamma)$.

$$
\operatorname{Br}(S \rightarrow \gamma \gamma) \approx\left(\frac{\alpha}{\alpha_{s}}\right)^{2} \frac{\left(3_{\mathrm{c}} Q_{T}^{2}\right)^{2}}{2} \approx 0.0070,
$$

where we substituted $Q_{T}=2 / 3$ and $\alpha=1 / 125 .{ }^{1}$ Finally, we obtain:

$$
\sigma_{p p \rightarrow S X} \cdot \operatorname{Br}(S \rightarrow \gamma \gamma) \approx 0.28 \mathrm{fb}
$$

Experimental data provides a value approximately 36 times larger:

$$
\left[\sigma_{p p \rightarrow S X} \cdot \operatorname{Br}(S \rightarrow \gamma \gamma)\right]_{\text {exp }} \approx 10 \mathrm{fb} .
$$

This value follows from the the fact that both collaborations see about 15 events with $3 \mathrm{fb}^{-1}$ luminosity collected by at $13 \mathrm{TeV}$ and effectivity of $\gamma \gamma$ registration $\varepsilon \approx 0.5$ [1].

In order to reproduce experimental result (11) we should suppose that six $T$-quarks exist. In this case $\operatorname{Br}(S \rightarrow \gamma \gamma)$ remains the same, while the cross section of $S$ production should be multiplied by the same factor 36 , and (11) is reproduced. ${ }^{2}$

However, unappealing multiplication of the number of $T$-quarks can be avoided by diminishing $T$ quark mass and increasing the value of $\lambda_{T}$. The experimental result is reproduced for $m_{T}=400 \mathrm{GeV}$ and $\lambda_{T}=2.5 .^{3}$ Curves of constant $\sigma_{p p \rightarrow S X} \cdot \operatorname{Br}(S \rightarrow \gamma \gamma)$ are shown in Figure 1 on $\left(\lambda_{T}, m_{T}\right)$ plot.

In the rest of this section we consider the model with one additional quark $T$ and $m_{T}=$ $400 \mathrm{GeV}, \lambda_{T}=2.5$. As it was shown above, this model can explain the diphoton excess but let us check that other existing bounds are not violated.

It is natural to suppose that $T$-quark mixes with $u$-, $c$-, and $t$-quark which makes it unstable. To avoid LHC Run 1 bounds on $m_{T}$ following from the search of the decays $T \rightarrow W b, T \rightarrow Z t$ and $T \rightarrow H t$ [8-10] which exclude $T$-quark with mass below $700 \mathrm{GeV}$, we suppose that $T-t$ mixture is

\footnotetext{
${ }^{1}$ Fine structure constant is substituted by its running value at $q^{2}=m_{S}^{2}$.

${ }^{2}$ The experimental result (11) will be reproduced if at $1 \mathrm{TeV}$ scale we have a "mirror image" of the Standard Model with three vector-like generations.

${ }^{3}$ As far as $\lambda_{T}^{2} / 4 \pi$ is a parameter of perturbation theory, this value of $\lambda_{T}$ is close to the maximum allowed value in order for the perturbation theory to make sense.
} 
small, and $T$-quark mixing with $u$ - and $c$-quarks dominates [11]. Though since we want the mass of $T$-quark to be just above the threshold of $S \rightarrow T T$ decay the mixing with the first two generations should not be too large in order to prevent the diminishing of $\operatorname{Br}(S \rightarrow \gamma \gamma)$ due to $S \rightarrow T T^{*} \rightarrow T W q$ decays.

Since at $\sqrt{s}=8 \mathrm{TeV}$ the gluon-gluon luminosity is 4.6 times smaller that at $\sqrt{s}=13 \mathrm{TeV}$, the CMS bound from Run $1\left[\sigma_{p p \rightarrow S X} \operatorname{Br}(S \rightarrow \gamma \gamma)\right]_{8 \mathrm{TeV}}<1.5 \mathrm{fb}$ [12] is (almost) not violated.

Concerning $S$ decays, let us note that the dominant $S \rightarrow g g$ decay is hidden by the two jets background produced by strong interactions. At $8 \mathrm{TeV}$ LHC energy the following upper bound was obtained [13]:

$$
\left[\sigma_{p p \rightarrow S X} \cdot \operatorname{Br}(S \rightarrow g g)\right]_{8 \mathrm{TeV}}^{\exp }<30 \mathrm{pb} .
$$

while in the considered model $\left[\sigma_{p p \rightarrow S X}\right]_{8 \mathrm{TeV}}^{\text {theor }} \approx 0.39 \mathrm{pb}$.

Three modes of $S$ decays to neutral vector bosons do exist and have the following hierarchy $\Gamma_{S \rightarrow \gamma \gamma}: \Gamma_{S \rightarrow Z \gamma}: \Gamma_{S \rightarrow Z Z}=1: 2\left(s_{W} / c_{W}\right)^{2}:\left(s_{W} / c_{W}\right)^{4}$, where $s_{W}\left(c_{W}\right)$ is the sine (cosine) of electroweak mixing angle (here we suppose that mixing of $S$ with Higgs doublet is negligible). Thus if the existence of $S$ will be confirmed by new data, $S \rightarrow \gamma Z$ and $S \rightarrow Z Z$ decays should be also looked for.

Scalar $S$ can mix with the Standard Model Higgs boson due to renormalizable interaction term $\mu \Phi^{\dagger} \Phi S$, where $\Phi$ is the Higgs isodoublet. Such an extension of the Standard Model was studied in our recent paper [14]. Due to doublet admixture there are tree level decays $S \rightarrow W W, Z Z, t \bar{t}$ and $h h$, where $h$ is the $125 \mathrm{GeV}$ Higgs boson. According to Eqs. (16)-(20) from [14], the sum of these widths equals approximately $\sin ^{2} \alpha \cdot m_{S}^{3} / 8 \pi v_{\Phi}^{2} \approx \sin ^{2} \alpha \cdot 300 \mathrm{GeV}$, where $\alpha$ is the mixing angle, and $v_{\Phi}=246 \mathrm{GeV}$ is the Higgs vev. Ratio of partial widths at small $\alpha$ is $\Gamma_{S \rightarrow W W}: \Gamma_{S \rightarrow Z Z}: \Gamma_{S \rightarrow h h} \approx 2: 1: 1$. As a result, $S$ width grows and $\operatorname{Br}(S \rightarrow \gamma \gamma)$ diminishes correspondingly.

To reproduce experimental result (11) and in order not to violate bounds from searches in these modes, the mixing angle $\alpha$ should be small enough. For example, for $\sin \alpha<1 / 150$ we obtain at most $12 \mathrm{MeV}$ (or 11\%) increase of the width of $S$, which is acceptable. It corresponds $|\mu|<15 \mathrm{GeV}$ which is not unnaturally small.

Let us check if for such mixing angle $S \rightarrow Z Z$ decays (due to doublet admixture) do not exceed experimental bounds obtained at the LHC. In the considered model

$$
\left[\sigma_{p p \rightarrow S X} \cdot \operatorname{Br}(S \rightarrow Z Z)\right]_{13 \mathrm{TeV}}<33 \mathrm{fb},
$$

well below experimental upper bound which, according to Fig. 11 from [15], equals $4 \mathrm{fb} /(\mathrm{Br}(Z \rightarrow$ $4 \ell))^{2}=400 \mathrm{fb}$ at $2 \sigma$ (see also [16]).

For $8 \mathrm{TeV}$ (taking into account energy dependence of the $K$-factor)

$$
\left[\sigma_{p p \rightarrow S X} \cdot \operatorname{Br}(S \rightarrow Z Z)\right]_{8 \mathrm{TeV}}<9.0 \mathrm{fb},
$$

while the experimental upper bound is $60 \mathrm{fb}$ (Fig. 12 from [17]).

More stringent upper bound comes from the search of $S \rightarrow h h$ decays [18] and equals $40 \mathrm{fb}$, while in our case the cross section does not exceed $10 \mathrm{fb}$.

Let us say a few words about future prospects. If the existence of $S$ will be confirmed with larger statistics at the LHC, then it can be studied at $e^{+} e^{-}$-colliders as well. For the cross section of $S$ production in photon fusion, according to [19, Eq. (48.47)], [20], we have:

$$
\sigma_{e e \rightarrow e e S}(s)=\frac{8 \alpha^{2}}{m_{S}^{3}} \Gamma_{S \rightarrow \gamma \gamma}\left[f\left(\frac{m_{S}^{2}}{s}\right)\left(\ln \left(\frac{m_{T}^{2} s}{m_{e}^{2} m_{S}^{2}}\right)-1\right)^{2}-\frac{1}{3} \ln ^{3}\left(\frac{s}{m_{S}^{2}}\right)\right],
$$




$$
f(z)=\left(1+\frac{1}{2} z\right)^{2} \ln \frac{1}{z}-\frac{1}{2}(1-z)(3+z),
$$

and $\Gamma_{S \rightarrow \gamma \gamma}$ is given in Eq. (8). At $e^{+} e^{-}$collider CLIC with $s=(3 \mathrm{TeV})^{2}$ for $\lambda_{T}=2.5$ and $m_{T}=$ $400 \mathrm{GeV}$ we obtain

$$
\sigma_{e e \rightarrow e e S}^{\mathrm{CLIC}} \approx 0.46 \mathrm{fb} .
$$

With projected CLIC luminosity $L=6 \cdot 10^{34} /\left(\mathrm{cm}^{2} \cdot \mathrm{sec}\right)[19, \mathrm{p} .393]$, during one accelerator year $\left(t=10^{7} \mathrm{sec}\right)$ about $300 \mathrm{~S}$ resonances should be produced.

\section{Leptophilic $S$}

$S$ production in $\gamma \gamma$ fusion will be analyzed in this section (see also [21-23]).

Let us suppose that heavy leptons $L_{i}$ which couple to $S$ have electric charges $Q_{L}$, and there are $N$ such degenerate leptons. The lagrangian is similar to that of the heavy quarks case (1):

$$
\Delta \mathcal{L}=\frac{1}{2}\left(\partial_{\mu} S\right)^{2}-\frac{1}{2} m_{S}^{2} S^{2}+\bar{L}_{i} \gamma_{\mu}\left(\partial_{\mu}-i g^{\prime} \frac{Y_{L}}{2} B_{\mu}\right) L_{i}+m_{L} \bar{L}_{i} L_{i}+\lambda_{L} \bar{L}_{i} L_{i} S,
$$

where we assume equal lepton masses and $S$ couplings. For $S \rightarrow \gamma \gamma$ width we obtain:

$$
\Gamma_{S \rightarrow \gamma \gamma}=\left(\frac{\alpha}{3 \pi}\right)^{2}\left(N Q_{L}^{2}\right)^{2} \frac{m_{S}^{3} \lambda_{L}^{2}}{16 \pi m_{L}^{2}}|F(\beta)|^{2}, \beta=\left(\frac{2 m_{L}}{m_{S}}\right)^{2} .
$$

Production of $S$ at the LHC occurs through fusion of two virtual photons emitted by quarks which reside in the colliding protons. Let us estimate the production cross section. For the partonic cross section we get:

$$
\sigma_{q_{1} q_{2} \rightarrow q_{1} q_{2} S}^{(\gamma \gamma)}(\hat{s})=\frac{8 \alpha^{2}}{m_{S}^{3}} e_{1}^{2} e_{2}^{2} \Gamma_{S \rightarrow \gamma \gamma}\left[f\left(\frac{m_{S}^{2}}{\hat{s}}\right)\left(\ln \left(\frac{m_{L}^{2} \hat{s}}{\Lambda_{\mathrm{QCD}}^{2} m_{S}^{2}}\right)-1\right)^{2}-\frac{1}{3} \ln ^{3}\left(\frac{\hat{s}}{m_{S}^{2}}\right)\right],
$$

where $e_{1}, e_{2}$ are charges of the quarks, $\hat{s}=x_{1} x_{2} s \equiv \tau s$, and $f(z)$ is given by (16). We should multiply (20) by PDFs and integrate over $x_{1}$ and $x_{2}$ :

$$
\begin{gathered}
\sigma_{p p \rightarrow S X}^{(\gamma \gamma)}(s)=\sum_{q_{1}, q_{2}} \int_{m_{S}^{2} / s}^{1} \sigma_{q_{1} q_{2} \rightarrow q_{1} q_{2} S}^{(\gamma \gamma)}(\tau s) d \tau \cdot s \cdot \frac{d L_{q_{1} q_{2}}}{d \hat{s}}\left(Q^{2}, \tau\right) . \\
\frac{d L_{q_{1} q_{2}}}{d \hat{s}}\left(Q^{2}, \tau\right)=\frac{1}{s} \int_{\ln \sqrt{\tau}}^{-\ln \sqrt{\tau}} q_{1}\left(x_{1}, Q^{2}\right) q_{2}\left(x_{2}, Q^{2}\right) d y,
\end{gathered}
$$

$x_{1}=\sqrt{\tau} \mathrm{e}^{y}, x_{2}=\sqrt{\tau} \mathrm{e}^{-y}$. We take $Q^{2}=m_{S}^{2}$ and use PDFs from [4].

Cross sections in the case of one heavy lepton with charge $Q_{L}=1$, Yukawa coupling constant $\lambda_{L}=2$ and mass $m_{L}=400 \mathrm{GeV}$ are shown in Table 1 . For $\Lambda_{\mathrm{QCD}}=300 \mathrm{MeV}$ and $\sqrt{s}=13 \mathrm{TeV}$ we get $\sigma_{p p \rightarrow S X}^{(\gamma \gamma)} \approx 11 \mathrm{ab},{ }^{4}$ while the experimental result (11) is three orders of magnitude larger. We come to the conclusion that $\sum N Q_{L}^{2} \approx 30$ is needed: we need either 30 leptons with unit charges, or one lepton with charge 6 , or several multicharged leptons. ${ }^{5}$

\footnotetext{
${ }^{4}$ According to Eq. (12) from the recent paper [24], this cross section equals 25 ab.

${ }^{5}$ If $\sigma_{p p \rightarrow S X}^{(\gamma \gamma)}=25 \mathrm{ab}$, then 30 should be replaced with 20 .
} 
Table 1. Cross sections (in ab) for double photon production in the leptophilic model for different values of $\Lambda_{\mathrm{QCD}}$ and proton collision energies.

\begin{tabular}{|c|c|c|c|c|}
\hline & \multicolumn{3}{|c|}{$\Lambda_{\mathrm{QCD}}, \mathrm{GeV}$} \\
\hline & & 0.1 & 0.3 & 1.0 \\
\hline & 7 & 2.5 & 1.9 & 1.3 \\
\hline & 8 & 3.8 & 2.9 & 2.0 \\
\hline 5 & 13 & 15 & 11 & 7.8 \\
\hline
\end{tabular}

It is natural to suppose that leptons with charge one mix with the Standard Model leptons and become unstable. Search for such particles was performed at the LHC, and the lower bound $m_{L}>$ $170 \mathrm{GeV}$ was obtained [25]. See also [26], where bounds on masses and mixings of $L$ are discussed. For masses above $200 \mathrm{GeV}$ the existence of $L$ is still relatively unconstrained.

Cross section for quasielastic $S$ production can be estimated with the help of the following equation:

$$
\sigma_{p p \rightarrow p p S}=\frac{8 \alpha^{2}}{m_{S}^{3}} \Gamma_{S \rightarrow \gamma \gamma}\left[f\left(\frac{m_{S}^{2}}{s}\right)\left(\ln \left(\frac{s}{m_{S}^{2}}\right)-1\right)^{2}-\frac{1}{3} \ln ^{3}\left(\frac{s}{m_{S}^{2}}\right)\right] .
$$

For $\sqrt{s}=13 \mathrm{TeV}, \lambda_{L}=2$ and $m_{L}=400 \mathrm{GeV}$ it equals $4.1 \mathrm{ab}^{6}$

\section{Conclusions}

We analyze the possibility that the enhancement at $750 \mathrm{GeV}$ diphoton invariant mass observed by ATLAS and CMS is due to decays of a new scalar $S$. We found that production of $S$ in gluon fusion in a minimal model with one additional heavy Dirac quark $T$ can have value of $\sigma_{p p \rightarrow S X} \cdot \operatorname{Br}(S \rightarrow \gamma \gamma)$ compatible with data. An upper bound on the mixing of $S$ with $h(125)$ is obtained. If heavy leptons $L$ are introduced instead of $T$, then $S$ can be produced at LHC in photon fusion, however, in order to reproduce experimental data many leptons $L_{i}$ are needed and/or they should be multicharged.

S. G. is partially supported by MK-4234.2015.2 and by RFBR under grants 16-32-60115, 14-0200995, and 16-02-00342. S. G. is also grateful to Dynasty Foundation for the support.

\section{References}

[1] The ATLAS collaboration, ATLAS-CONF-2015-081 (2015)

[2] The CMS collaboration, CMS-PAS-EXO-15-004 (2015)

[3] Godunov S.I., Rozanov A.N., Vysotsky M.I., Zhemchugov E.V., JETP Lett. 103, 635 (2016)

[4] Harland-Lang L. A., Martin A. D., Motylinkski P., Thorne R. S., Eur. Phys. J. C 75, 204 (2015)

[5] Baglio J., Djouadi A., JHEP 1103, 055 (2011)

[6] Harlander R. V., Kilgol W., Phys. Rev. Lett. 88, 201801 (2002)

[7] Buckley M. R., Eur. Phys. J. C 76, 345 (2016)

[8] The ATLAS collaboration, JHEP 1510, 150 (2015)

[9] The ATLAS collaboration, JHEP 1508, 105 (2015)

[10] The CMS collaboration, Phys. Rev. D 93, 012003 (2016)

[11] Buchkremer M., Proc. of 49th Rencontres de Moriond on Electroweak Interactions and Unified Theories, 519 (2014)

\footnotetext{
${ }^{6}$ According to Eq. (24) from [24], quasielastic cross section is two times smaller.
} 
[12] The CMS collaboration, Phys. Lett. B 750, 494 (2015)

[13] The ATLAS collaboration, Phys. Rev. D 91, 052007 (2015)

[14] Godunov S. I., Rozanov A. N., Vysotsky M. I., Zhemchugov E. V., Eur. Phys. J. C 76, 1 (2016)

[15] The ATLAS collaboration, ATLAS-CONF-2015-59.

[16] Franceschini R. et. al., JHEP 1603, 144 (2016)

[17] The ATLAS collaboration, Eur. Phys. J. C 76, 45 (2016)

[18] The ATLAS collaboration, Phys. Rev. D 92, 092004 (2015)

[19] Olive K. A. et al. (Particle Data Group), Chin. Phys. C 38, 090001 (2014)

[20] Budnev V. M., Ginzburg I. F., Meledin G. V., Serbo V. G., Phys. Reports 15, 181 (1975)

[21] Fichet S., vob Gersdorff G., and Royon C., Phys. Rev. D 93, 075031 (2016)

[22] Csaki C., Hubisz J., and Terning J., Phys. Rev. D 93, 035002 (2016)

[23] Pilaftis A., Phys. Rev. D 93, 015017 (2016)

[24] Harland-Lang L. A., Khoze V. A., Ryskin M. G., JHEP 1603, 182 (2016)

[25] The ATLAS collaboration, JHEP 1509, 108 (2015)

[26] A. Djouadi, J. Ellis, R. Godbole, J. Quevillon, arXiv:1601.03696. 